\title{
Maintenance of Integrity of Upper Facet Joints during Simulated Percutaneous Pedicle Screw Insertion Using 2D versus 3D Planning
}

\author{
Eleftherios Archavlis ${ }^{1} \quad$ Florian Ringel $^{1} \quad$ Sven Kantelhardt ${ }^{1}$ \\ ${ }^{1}$ Department of Neurosurgery, University of Mainz, Mainz, Germany \\ Address for correspondence Eleftherios Archavlis, Department of \\ Neurosurgery, University of Mainz, Langenbeckstr. 1, Mainz 55131, \\ J Neurol Surg A 2019;80:269-276. \\ Germany (e-mail: neurosurgery@t-online.de).
}

\begin{abstract}
Keywords

- pedicle screws

- 3D simulation study

- minimally invasive

- facet joint violation

- robotic

Background No studies have directly and quantitatively compared two-dimensional (2D) and three-dimensional (3D) planning as applied during conventional percutaneous or navigated percutaneous pedicle screw placement.

Study Aims This lumbar pedicle-based stabilization simulation study aimed to investigate the risk of upper facet joint violation (FJV) during posterior percutaneous pedicle screw placement with conventional 2D planning of screw implantation (as a model for fluoroscopically guided screws) compared with 3D planning (as used with navigation techniques).

Methods The placement of monosegmental lumbar pedicle screws using the data sets of 250 consecutive patients was simulated. Conventional surgery (using 2D fluoroscopic images anteroposterior and lateral view) was compared with screw placement using the 3D reconstruction of the planning mode of the same software. Results The 2D planning resulted in 140 upper FJVs (28\% of cases), whereas 3D planning resulted in only 24 upper FJVs ( $4.8 \%$ of cases) $(p<0.05)$. Among those spinal segments with severe facet joint arthropathy, Pathria grades 3 and 4, FJV was significantly higher $(p<0.05)$ in the 2D-planned screws $(64.7 \%)$ than in the 3Dplanned screws (11.2\%). A more lateral (mean distance: $3.5 \mathrm{~mm}$ ) and inferior (mean distance: $2.5 \mathrm{~mm}$ ) offset of the pedicle entry point and a larger medial angulation of the trajectory (mean angle: 9 degrees) were observed for the 3D-planned screws at all levels.

Conclusion This study demonstrates that the use of $2 \mathrm{D}$ planning is associated with a higher risk of upper FJV than when a 3D imaging data set is used. Using a more lateral and inferior entry point for fluoroscopically guided pedicle screws could reduce the rate of FJV in percutaneous pedicle screw placement.
\end{abstract}

\section{Introduction}

Lumbar pedicle screw placement can be challenging due to the geometric complexity of the pedicles and the proximity of the facet joints. Percutaneous instrumentations can be especially demanding due to the limited visibility of anatomical landmarks and a restricted working space. ${ }^{1}$ Pedicle screws require good accuracy to achieve satisfactory place-

received

July 31,2018

accepted after revision

October 17, 2018

published online

April 24, 2019

ment without violation of pedicle walls but also to maintain the integrity of the superior facet joints that will not be included in the fusion.

However, violation of the superior facet joints is a frequent problem that could lead to persisting symptoms and adjacent facet degeneration associated with higher reoperation rates and diminished improvement in quality of life. ${ }^{2}$ Rates reported range from 4 to $100 \%$ and are especially high in minimally

(c) 2019 Georg Thieme Verlag KG Stuttgart . New York
DOI https://doi.org/ 10.1055/s-0039-1681042. ISSN 2193-6315. 
invasive percutaneous procedures guided by two-dimensional (2D) fluoroscopy. ${ }^{3,4}$ In a previous patient study we compared three different pedicle screw insertion 2D techniques with regard to their frequency of facet joint violations (FJVs) and revealed a significant lack of accuracy with the conventional minimal invasive percutaneous procedure. ${ }^{5}$ The comparison of a percutaneous robotic, percutaneous fluoroscopic, or standard open technique for pedicle screw implantation revealed rates of $5 \%, 22 \%$, and $6 \%$ for upper FJVs, respectively.

Computer-assisted navigation technologies were developed for pedicle screw placement to improve implantation accuracy. ${ }^{6}$ Systems for conventional or robotic image guidance generally provide software tools for three-dimensional (3D) planning of implant positions and some form of intraoperative guidance (image based or physical by holding drill guides, etc.). Clinical studies on these techniques demonstrated favorable radiologic results compared with solely freehand techniques. ${ }^{6-8}$

In a clinical evaluation using a robotic navigation system, we recently showed that the application of image guidance and 3D planning could help the surgeon perform pedicle screw placement sparing upper facet joints. ${ }^{5,9}$ However, the question remains whether the effect can be attributed rather to the intraoperative guidance than to improved visibility of anatomical structures during 3D planning of implant positions. To date, no studies have directly and quantitatively compared 2D and 3D planning as applied during conventional percutaneous or navigated percutaneous pedicle screw placement.
This experimental study was conducted to investigate whether the mode of planning (using 2D or 3D imaging tools) alone could have a significant effect on implant positions, and whether 3D visualization during planning might help spare the adjacent upper facet joint in pedicle screw placement. Differences between the two techniques in the location of the entry point and trajectory to the pedicle axis were assessed.

\section{Methods}

\section{Experimental Setup}

We performed a simulated instrumentation on a software reconstruction. Informed consent and ethical approval were waived by the corresponding ethical board, based on the nonclinical nature of this study.

Imaging data of 250 consecutive patients who underwent lumbar fixation for degenerative spine disease at our institution between 2012 and 2016, and who met the inclusion criteria were included in the study. - Fig. 1 depicts the inclusion criteria. Patients with infection, tumor, revision surgery, congenital deformity, and/or trauma or those who did not succeed in registration of the navigation software due to technical problems (e.g., unsatisfactory imaging of the lumbar levels involved, metal artifacts from prior surgeries causing noise in 3D reconstruction images) were excluded.

The patients' imaging data sets $(1 \mathrm{~mm}$ thickness axial computed tomography [CT] scans from L2-S1, Aquilion RXL

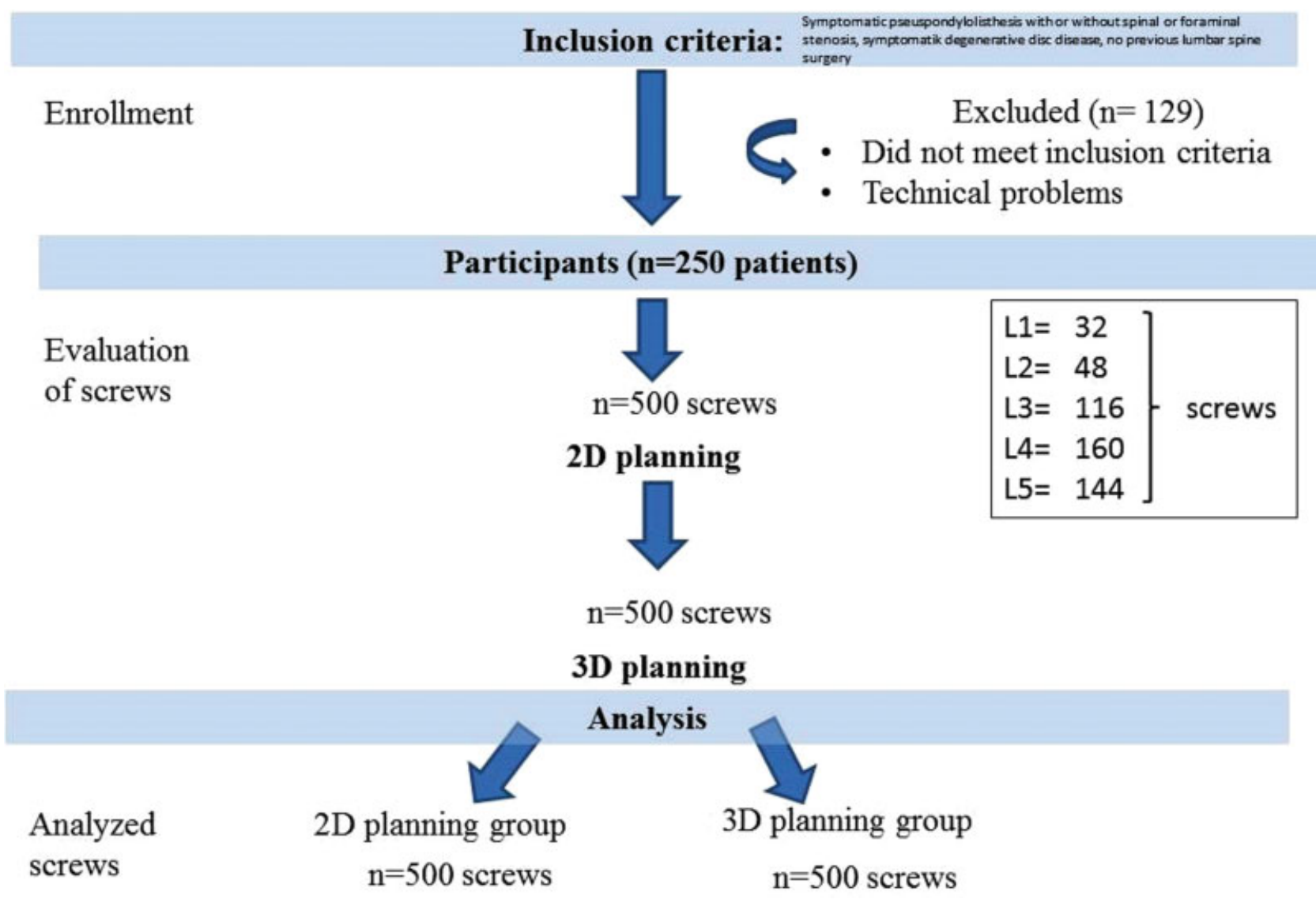

Fig. 1 Trial flowchart. 
multislice scanner, Toshiba, Zoetermeer, Netherlands) were processed with Renaissance planning software (Mazor Robotics, Caesarea, Israel). The software provided 2D (anteroposterior $[\mathrm{AP}]$ and lateral translucent $2 \mathrm{D}$ reconstructions of the CT images resembling fluoroscopy images) and 3D visualization of the CT (CT cuts in axial, sagittal, and coronal reconstructions) of the lumbar spine. First, all data sets underwent conventional pedicle screw placement using 2D fluoroscopic images in AP and lateral views (2D group). We subsequently performed screw placement using the 3D reconstruction and planning mode of the same spinal navigation-planning software (3D group). From the axial source images, sagittal and coronal images were reconstructed. All levels between L1and L5 were virtually instrumented in each data set. All simulated procedures were performed by the same surgeon, experienced in both techniques. After completion of the simulation, all data sets were subsequently assessed by two surgeons blinded to the applied technique.

\section{Planning of Pedicle Screw and Assessment of Entry Point and Trajectory}

At first, the planning surgeon positioned the screws using AP and lateral radiographic images only (2D group) as used in fluoroscopy-guided instrumentation. After that, the surgeon simulated the pedicle screw using the complete 3D planning tools of the software (3D group) as applied in navigation and robot-guided procedures. For both techniques, the surgeon's aim was to position the screw with a 1 -mm safety margin to all pedicle walls and respecting the superior articular process and articular facet with a safety margin of $2 \mathrm{~mm}$ to the screw shaft and screwhead. The priority was focused on respecting the pedicle walls and placing pedicle screws as accurately as possible.
In the 2D group, pedicle screws were planned to be inserted according to the method described by Weinstein et al using an entry point at the "nape of the neck" of the superior articular process and an inward trajectory. ${ }^{10}$ On the AP radiograph of the lumbar spine, this point corresponds to the lateral ridge of the pedicle projection ( - Fig. 2). From this entry point the fictive pedicle probe is placed in the center of the pedicle, and the trajectory is controlled in the lateral view to confirm that the fictive probe reaches the pedicle-vertebral body junction. Keeping this sagittal angulation, the definite screw position is planned with the distal end of the trajectory placed in the anterior vertebral body and the middle part seen within the boundaries of the pedicle on the AP view but lateral to the medial pedicle wall.

In the 3D group, screw positions were reviewed and optimized in all three planes (axial, sagittal, and coronal) (-Fig. 3).

Finally, differences of the screw entry points were quantified. The deviation of the 3D entry point was measured from the 2D entry point as lateral and craniocaudal deviation in millimeters (-Fig. 2). The convergence angle was also compared between the two groups.

\section{Evaluation of Pedicle Screws with Respect to Facet Joints}

The 3D planning software was also used to evaluate resulting FJV from L1-L2 to L4-L5. This was performed by two surgeons independently who were blinded to the applied planning mode. The simulated FJV included not only the trajectory but also the screw head/tulip position and the screw diameter of $6 \mathrm{~mm}$.

Abutment of the polyaxial screw head on the dorsal surface of the facet joint and interposition of screw threads between the superior and inferior articular processes of the facet joint were classified as an FJV.

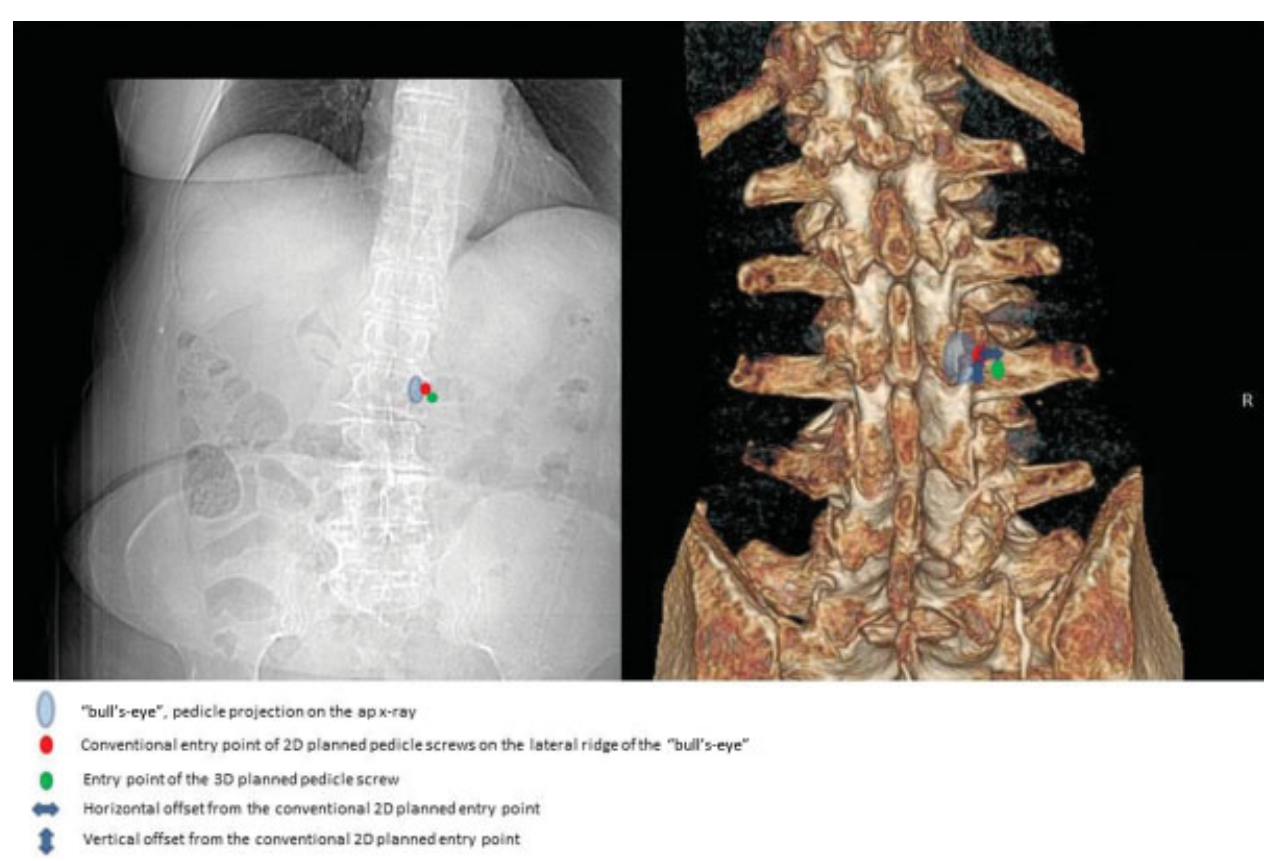

Fig. 2 Virtual lumbar pedicle screw placement using the anteroposterior radiograph for the 2D technique und the three dimensionally reconstructed model for the $3 \mathrm{D}$ technique. 


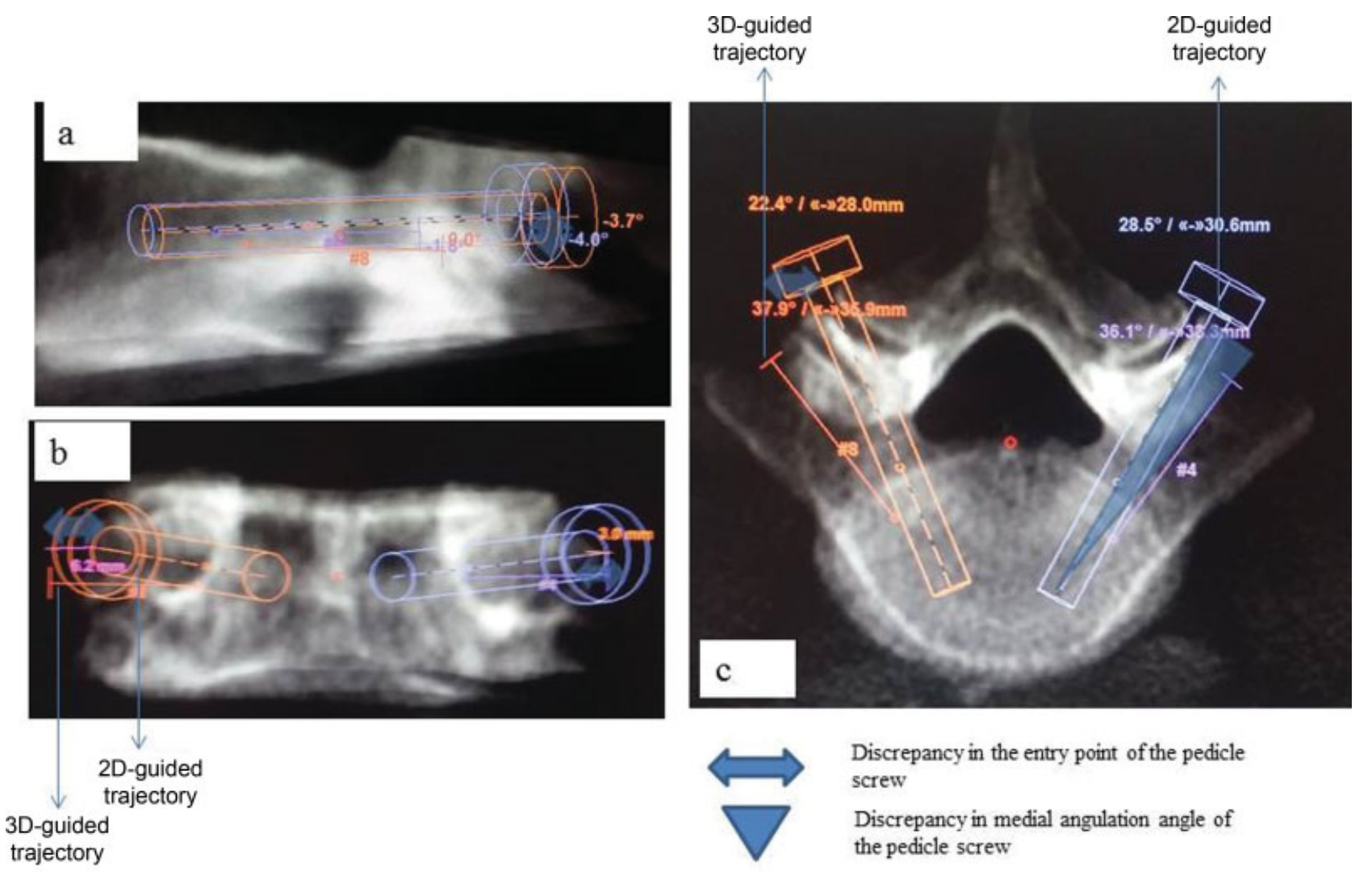

Fig. 3 Measurements of the radiologic parameters of the two planning techniques presented in a single case: (a) vertical offset, (b) horizontal offset of the 3D entry point from the 2D-placed pedicle screw, and (c) transverse angle on the reconstructed axial image with greater angulation angle in the 3D-placed trajectory.

Additionally, facet joint osteoarthritis was graded on a 4point scale according to Pathria. ${ }^{11,12}$ We then analyzed whether the degree of structural facet disease represented a risk factor for upper FJV.

\section{Evaluation of Pedicle Screws in Respect to Pedicle Walls}

Screw placement was considered correct if the screw was completely surrounded by the pedicular cortex. An incorrect screw position was categorized as cortical encroachment if the pedicle cortex could not be visualized or as frank penetration when the screw was outside the pedicular boundaries.

\section{Evaluation of Time Required for the Planning}

Apart from accuracy data, the time required for planning of the procedures was recorded.

\section{Statistical Analysis}

All data were analyzed using SPSS v.22.0 (IBM Inc., Armonk, New York, United States). For each technique, we evaluated the overall frequency of FJV and the relative risk of violation caused by facet joint osteoarthritis. Then we performed two comparative analyses. First, we compared the overall frequency of FJVs by $2 \mathrm{D}$ - versus $3 \mathrm{D}$-planned screws in the entire population. Second, the same comparison was performed for the subset of patients with severe facet joint osteoarthritis (Pathria grades 3 and 4). The frequency of facet joint osteoarthritis was com- pared using the Fisher exact test (for independent proportions) or the McNemar test (for paired proportions). The level of significance was set at $p<0.05$ for all statistical analyses.

\section{Results}

CT scans of 379 consecutive patients were used in this study. Overall, 250 patients fulfilled the selection criteria; their data sets were included. We performed virtual instrumentation in each data set, so a total number of 500 adjacent cranial facet joints and pedicle screws could be assessed. The trial flowchart in - Fig. 1 offers an overview of the steps performed. Imaging data originated from 153 women and 97 men with an average age of 57 years (range: $35-81$ years). There were 330 facet joints with an osteoarthritis grade $1 / 2$ und 170 with grade $3 / 4$ among the 500 assessed screw insertion sites.

\section{Comparison of 2D- versus 3D-Planned Percutaneous Pedicle Screw Insertion for Upper Facet Joint Violation Rates}

Screw trajectories were evaluated bilaterally, for a total of 500 possible screw insertion sites for each type of technique. Overall, 2D-planned screws violated upper facet joints in $28 \%$ (140/500 screws). Among the 170 facet joints with an osteoarthritis grade $3 / 4$, the 2D-planned screws violated the facet joint in 110 (93.5\%). Among the 330 possible screw insertion sites with low-grade osteoarthritis grade $1 / 2$, the 
2D-planned screws violated the upper facet joint in only 30 (9.1\%). Therefore, the frequency of FJVs by 2D-planned screws was significantly higher in high-grade facet joint osteoarthritis than in low-grade osteoarthritis $(p<0.001)$, with a relative risk of 10.27 .

The 3D-planned screws violated the upper facet joints in $4.8 \%$ of cases (24/500 screws). Among the 170 possible screw insertion sites with facet joint osteoarthritis 3/4, the 3Dplanned screws violated the facet joint in 19 (11.1\%). Among the 330 possible screw insertion sites with low-grade osteoarthritis grade $1 / 2$, the $3 \mathrm{D}$-planned screws violated the upper facet joint in only 5 (1.5\%). Therefore, the frequency of FJVs by 3D-planned screws was also higher in high-grade facet joint osteoarthritis than in low-grade osteoarthritis $(p<0.001)$, with a relative risk of 7.4. The difference in frequency of upper FJVs, 28\% in 2D-planned screws versus $4.8 \%$ in 3D-planned screws, was statistically significant $(p<0.001$ ) ( - Table 1 ). Among the 170 possible insertion sites with facet joint osteoarthritis grade $3 / 4$, FJV was significantly lower with 3D-planned screws (19 violations, $11.1 \%$ ) than with 2D-planned screws (110 violations [64.7\%])

\section{Comparison of 2D- versus 3D-Planned Percutaneous Pedicle Screw Insertion for Pedicle Wall Violation \\ Rates}

In terms of pedicle wall integrity, no statistically significant difference between the two groups was found. Among the 500 pedicle screws simulated in this study, 463 (92.6\%) of the $2 \mathrm{D}$ group and all the screws of the $3 \mathrm{D}$ group were interpreted as correctly inserted within the pedicle. Cortical encroachment was found for 35 screws (7\%), and frank penetration (> $2 \mathrm{~mm}$ ) for $2(0.4 \%)$ of the $2 \mathrm{D}$ group.

\section{Comparison of 2D- versus 3D-Planned Percutaneous Pedicle Screw Insertion for Entry Points and Trajectories}

The difference between the entry point locations for the 2Dand 3D-planned screws are depicted in - Tables 2 and 3. A more lateral (mean distance: $3 \mathrm{~mm}$ ) and inferior (mean distance: $2.5 \mathrm{~mm}$ ) offset of the pedicle entry point on the lateral ridge of the pedicle projection on the AP radiograph (-Table 2) and a larger medial angulation of the trajectory (mean angle: 9 degrees) were observed in the 3D group at all levels (-Table 3 ). No statistically significant difference was observed inside the $3 \mathrm{D}$ group between mild and severe degenerated facet joints.

\section{Time for Planning of Surgery}

In the statistical analysis, the mean time for the completion of the 3D planning was 23 minutes (95\% confidence interval [CI], 15-28 minutes), whereas the time spent in the 2D group was 12 minutes (95\% CI, 5-19 minutes). This difference was statistically significant $(p<0.05)$.

\section{Discussion}

The study presented here is the continuation of a previous retrospective report that assessed the performances of the 3D-planned and percutaneous robot-guided pedicle screw

Table 1 Upper facet joint violations by 2D- versus 3D-planned screws

\begin{tabular}{|l|l|l|l|}
\hline Variants & $\begin{array}{l}\text { Violations by } \\
\text { 2D-planned screws \% }(n)\end{array}$ & $\begin{array}{l}\text { Violations by } \\
\text { 3D-planned screws \% }\end{array}$ & $p^{\text {a }}$ \\
\hline Overall $(n=500)$ & $28(140)$ & $4.8(24)$ & $<0.0001$ \\
\hline $\begin{array}{l}\text { Facet joint osteoarthritis } \\
\text { Pathria grades 3 and 4 }(n=170)\end{array}$ & $65(110)$ & $11(19)$ & $<0.0001$ \\
\hline
\end{tabular}

${ }^{\mathrm{a}}$ McNemar test is used.

Table 2 Evaluation of horizontal and inferior vertical offsets from conventional entry point of 2D-planned screw after application of 3D planning technique for each level

\begin{tabular}{|c|c|c|c|c|c|c|}
\hline \multirow{3}{*}{ Level } & \multicolumn{2}{|c|}{ Horizontal offset, mm } & & \multicolumn{2}{|c|}{ Vertical offset, mm } & \multirow{3}{*}{$p$ value } \\
\hline & \multicolumn{2}{|l|}{ 3D } & \multirow[t]{2}{*}{$p$ value } & \multicolumn{2}{|l|}{ 3D } & \\
\hline & $\begin{array}{l}\text { Facet joint } \\
\text { osteoarthritis } \\
\text { Pathria } 1-2\end{array}$ & $\begin{array}{l}\text { Facet joint } \\
\text { osteoarthritis } \\
\text { Pathria } 3-4\end{array}$ & & $\begin{array}{l}\text { Facet joint } \\
\text { osteoarthritis } \\
\text { Pathria } 1-2\end{array}$ & $\begin{array}{l}\text { Facet joint } \\
\text { osteoarthritis } \\
\text { Pathria 3-4 }\end{array}$ & \\
\hline L5 & $3.5 \pm 1.5$ & $4.5 \pm 0.5$ & 0.722 & $3 \pm 1$ & $4 \pm 1$ & 0.722 \\
\hline L4 & $3.5 \pm 1$ & $4 \pm 1$ & 0.722 & $2.5 \pm 1$ & $3 \pm 1.5$ & 0.714 \\
\hline L3 & $3 \pm 1.5$ & $4 \pm 1$ & 0.722 & $2.5 \pm 0.5$ & $3.5 \pm 1$ & 0.714 \\
\hline $\mathrm{L} 2$ & $2.5 \pm 1.5$ & $3 \pm 2.5$ & 0.714 & $1.5 \pm 2$ & $2 \pm 1$ & 0.7 \\
\hline L1 & $2 \pm 1.5$ & $2.5 \pm 2.5$ & 0.8 & $1.5 \pm 2$ & $2 \pm 1$ & 0.7 \\
\hline
\end{tabular}

Data are presented as mean and standard deviation. 
Table 3 Evaluation of transverse medial angulation angle from conventional trajectory of 2D-planned screw after application of $3 \mathrm{D}$ planning technique for each level

\begin{tabular}{|l|l|l|l|}
\hline & \multicolumn{2}{|l|}{ Transverse angles, degrees } & \\
\hline Level & \multicolumn{2}{|l|}{ 3D } & $p$ value \\
\hline & $\begin{array}{l}\text { Facet joint } \\
\text { osteoarthritis } \\
\text { Pathria 1-2 }\end{array}$ & $\begin{array}{l}\text { Facet joint } \\
\text { osteoarthritis } \\
\text { Pathria 3-4 }\end{array}$ & \\
\hline L5 & $14 \pm 5$ & $16 \pm 6$ & 0.725 \\
\hline L4 & $10 \pm 7$ & $13 \pm 5$ & 0.696 \\
\hline L3 & $7 \pm 2$ & $8 \pm 3$ & 0.735 \\
\hline L2 & $5 \pm 3$ & $6 \pm 2$ & 0.730 \\
\hline L1 & $5 \pm 5$ & $4 \pm 6$ & 0.727 \\
\hline
\end{tabular}

Data are presented as mean and standard deviation.

placement when compared with a percutaneous fluoroscopic-guided freehand technique in clinical practice. ${ }^{5}$

\section{Impact of 3D Planning on Upper Facet Joint Violation Rates}

The results presented here indicate the pedicle screw accuracy in terms of the integrity of the upper facet joints is higher in the 3D-planned percutaneous pedicle screw simulation than in procedures where only 2D images are available. It appears that the 3D-guided technique leads to a more lateral and inferior entry point compared with the conventional 2D fluoroscopy-guided technique. This could be explained by the fact that the facet joints are only poorly visualized in the AP and lateral fluoroscopic view but rather in the oblique view, which is not popular among spine surgeons because orientation is very difficult. ${ }^{3}$

Our results showed that 3D planning reduced the rates of upper FJV in a simulation setting. Several studies reported on the incidence of FJV with percutaneously placed pedicle screws. ${ }^{13,14}$ Although many studies investigated the rate of pedicle perforations in percutaneous and/or navigated procedures, $<10 \%$ of these trials and most of them since 2010 report the results on upper FJV rates. ${ }^{3-5,8,13-20}$ The development of 3D navigation systems during the last few years and the increasing interest in the prevention of adjacentlevel disease due to increased spine instrumentations may be the reason for this new focus.

Facet joint abutment through the screw head is possible, but in clinical practice it is clearly associated with the insertion depth of the pedicle screw, a fact that can be modified intraoperatively according to the anatomical conditions.

According to our results, application of 3D planning tools moved the entry point more laterally and inferiorly. Thus surgeons need to modify the insertion technique of the percutaneous technique when based only on 2D fluoroscopic images. If surgeons are not equipped with navigation tools, robotic assistance, and 3D fluoroscopic devices, we recommend the following: (1) Plan the screws thoroughly according to the preoperative CT data. (2) Palpate the anatomy of the transverse and lateral facet through a mini open extension of the incision, avoiding penetration of the joint capsule. (3) Use a more lateral (mean distance: $3 \mathrm{~mm}$ ) and inferior (mean distance: $2.5 \mathrm{~mm}$ ) offset of the pedicle entry point on the lateral ridge of the pedicle projection on the AP radiograph. (4) Consider a sufficient medial angulation so the screw tip reaches the ventral third and also reaches the medial third of the vertebral body.

\section{Effect of Facet Degeneration}

Although many reports have proposed the optimal entry points for avoiding penetration of facet joints, these studies did not consider the technique of implantation and the degree of facet joint degeneration. ${ }^{21-23}$ Hypertrophy and osteophytes of the facet joints are observed with aging and degenerative disease. Thus changes of the entry points and angulation angle in situ in cases of open approach and in the conventional fluoroscopy images in cases of minimally invasive percutaneous approach may be necessary. However, until now no studies have compared entry points and trajectories of lumbar pedicle screws according to their 2D- or 3D-planned routes. Thus understanding the entry point and trajectories of pedicle screws is critical to avoid violation of upper unfused facet joints. According to our results, surgeons need to modify the insertion technique of the 2D-planned pedicle screws when considering inserting screws at the upper vertebra, especially in the degenerative lumbar spine, to maintain the integrity of the upper unfused facet joint. In fact, pedicle screw insertion during fluoroscopy-guided percutaneous surgery with a minimum of $3 \mathrm{~mm}$ lateral to the outer pedicle margin on the AP radiograph might be a good option. However, it is technically demanding and could lead to perforation of the lateral pedicle wall. Therefore, a 3D-guided technique as provided by robotic or image guidance could be considered an option if degenerative changes are severe.

\section{Clinical Significance of Upper Facet Violation}

FJV does not always cause clinical symptoms. But the violation of the most upper adjacent facet joint could increase the risk of facet arthritis. Lumbar fusion is reported to accelerate degenerative changes of the adjacent facet joints and disks. ${ }^{24}$ In addition, facet degeneration could be a significant factor in the occurrence of low back pain and adjacent-level disease, which is more prominent in the proximal adjacent segment. ${ }^{9}$ In a retrospective cohort study of 240 patients, FJV was independently associated with a higher reoperation rate and diminished improvement in quality of life. ${ }^{2}$ At 2-year follow-up, patients in the FJV group were less likely to make a significant improvement on the EQ-5D questionnaire $(p=0.041)$. Also, the reoperation rate in the FJV group was significantly higher than in the control group at 2 years (15.2\% versus $6.3 \%$, respectively; $p=0.024$ ) and 3 years (19.6\% versus $9.4 \% ; p=0.023$ ). Thus violation of the upper unfused facet joints by the pedicle screws might accelerate the degenerative process of the adjacent level. Hence the surgeon should (1) plan the screws preoperatively, and pay attention to morphological changes of the spine that could affect the entry point and trajectory; and (2) consider 
intraoperatively the inherent risk of FJV of the percutaneous 2D fluoroscopy-guided insertion technique.

The role of soft tissue violation surrounding the facet joints, causing capsular impingement, for example, has not been studied yet, and it is unclear whether it is promoting osteoarthritic changes.

\section{Time for Planning of Surgery}

We found that the process of 3D-based planning required significantly more time compared with conventional 2D planning ( $\sim 23$ versus 12 minutes). This is a drawback, of course, because time is scarce in clinical routine. However the 11 or 12 additional minutes may be well spent if they facilitate surgery and render the process safer. One single revision will quite outweigh several planning procedures. Furthermore, current 3D planning (and navigation) software generally allow archiving the preoperative plan. Although this is not yet obligatory for spinal instrumentation, it is mandatory in other fields like knee or hip replacement surgery and definitely helpful if questions arise later on.

\section{Pedicle Wall Integrity}

In this simulation study we found good accuracy of the pedicle screws as far as the pedicle wall integrity is concerned when using 2D planning during simulated surgery. Although we did not observe any pedicle wall violations in the 3D group, this difference was found to be statistically insignificant. Although published clinical series described a lower rate of pedicle violations, ${ }^{6-8,24,25}$ the failure to show this in our study might be attributed to the relatively low number of cases (250 patients). Or, more probable, the difference observed in clinical series was due to the intraoperative guidance provided by navigation systems rather than an improved planning tool.

\section{Usefulness of 3D Visualization for Educational Purposes}

The use of 3D monitoring systems in medical education offers the advantage of stereopsis and contributes to surgical training. ${ }^{26}$ In spine surgery, 3D visualization can be extremely useful for navigating complex deformities and improving anatomical understanding for training. Furthermore, 3D visualization software could be useful in understanding space interval and depth of implants in relation to spine structures. Medical students, medical support staff, and patients who are unfamiliar with the anatomy of the pathology can easily understand anatomical relationships with 3D models. And 3D printing is a further development and a growing transformative technology with a potentially wide range of applications in the field of spine surgery. Life-size 3D models can not only allow observation but also actual cutting and drilling using surgical instruments, which in turn could considerably enhance a surgeon's skill and improve risk management for complex surgical procedures.

\section{Limitations of the Study}

The presented study only assesses the planning process. Upon translation of the planning results into clinical practice, a certain number of additional FJVs will occur due to imprecise intraoperative positioning of screws. However, actual placement of screws into bone in experienced hands is not a completely radiographic procedure and is enabled through tactile feedback. The surgeon can palpate the anatomy of the transverse and lateral facet even in percutaneous procedures through a mini open extension of the incision and can merge the information gleaned from the fluoroscopy. Nevertheless, the difference observed here is relevant because clinical results could presumably worsen if only 2D fluoroscopic data are relied on. The application of a navigation system will also be beneficial at this stage of the operation.

Until recently the significance of upper FJVs was only hypothesized. ${ }^{9,24}$ In 2018, Levin et al presented the first clinical evidence highlighting the potential morbidity associated with FJV at the superior-most fusion level. ${ }^{2}$ Further studies are needed to prove that facet osteoarthritis through pedicle screw violation is a relevant clinical entity. Nevertheless, apart from this point, the data presented show that careful planning using 3D data may improve the precision of pedicle screw placement in general.

Another point is the applied software. Although several types of spinal navigation and planning software exist, every such tool can be prone to some errors, and results should eventually be verified in a clinical series. Especially those patients who had to be excluded from the study because of technical difficulties with the registration process might be problematic cases that are not covered by our results.

The screws in our study were all placed by a single surgeon, experienced in both conventional and navigated techniques. This makes the data more coherent, but of course it also depends on the individual capabilities of this surgeon. If the study was conducted by another individual with his or her set of experiences, the results might be different. This is a clear limitation because the reliability between more than one observer could not be assessed.

Although rapidly developing, spinal navigation and robotic surgical spinal technology have not achieved their full potential owing to some limitations. Cost effectiveness is a major issue that is not clarified in the literature. Other drawbacks to robotic surgery include the lack of tactile feedback to the surgeon and the bulkiness of the robotic equipment currently in use.

Nevertheless, regardless of the limitations just described, this is the first study in a consecutive group of patients to assess the value of $3 \mathrm{D}$ versus $2 \mathrm{D}$ planning quantitatively in regard to pedicle screw accuracy and adjacent facet joint integrity. The results presented here suggest that $3 \mathrm{D}$ reconstruction and planning is a useful tool for preoperative planning of pedicle screw positions.

\section{Conclusion}

This study demonstrates that the application of only 2D fluoroscopic images for pedicle screw insertion has a more inherent anatomical risk of upper FJV. Application of 3D planning tools moved the entry point more laterally and increased the rate of radiographically intact upper facet 
joints. Thus surgeons need to modify the insertion technique of the percutaneous technique when based on only 2D fluoroscopic images. In the presence of severe facet joint arthropathy, simulated placement of 3D-planned screws is significantly safer than the placement of 2D-planned screws.

Complementary prospective randomized in vivo studies could be performed to fully validate the results observed here in terms of accuracy, repeatability, and ergonomics.

\section{Financial Support}

No grants were received for this work. The authors have no personal or institutional financial interest in drugs, materials, or devices described in this submission.

\section{References}

1 Archavlis E, Carvi y Nievas M, Ulrich P. Preliminary results of minimally invasive decompression, TLIF and percutaneous pedicle screw insertion in stenotic spondylolisthesis with severe facet joint osteoarthritis. J Spine 2013;S5:4

2 Levin JM, Alentado VJ, Healy AT, Steinmetz MP, Benzel EC, Mroz TE. Superior segment facet joint violation during instrumented lumbar fusion is associated with higher reoperation rates and diminished improvement in quality of life. Clin Spine Surg 2018; 31(01):E36-E41

3 Knox JB, Dai JM III, Orchowski JR. Superior segment facet joint violation and cortical violation after minimally invasive pedicle screw placement. Spine J 2011;11(03):213-217

4 Patel RD, Graziano GP, Vanderhave KL, Patel AA, Gerling MC. Facet violation with the placement of percutaneous pedicle screws. Spine 2011;36(26):E1749-E1752

5 Archavlis E, Amr N, Kantelhardt SR, Giese A. Rates of upper facet joint violation in minimally invasive percutaneous and open instrumentation: a comparative cohort study of different insertion techniques. J Neurol Surg A Cent Eur Neurosurg 2018;79(01):1-8

6 Kantelhardt SR, Martinez R, Baerwinkel S, Burger R, Giese A, Rohde V. Perioperative course and accuracy of screw positioning in conventional, open robotic-guided and percutaneous robotic-guided, pedicle screw placement. Eur Spine J 2011;20(06):860-868

7 Kantelhardt SR, Keric N, Conrad J, et al. C-OnSite ${ }^{\circledR}$ for intraoperative 3D control of pedicular screw positions. Acta Neurochir (Wien) 2014;156(09):1799-1805

8 Ohba T, Ebata S, Fujita K, Sato H, Haro H. Percutaneous pedicle screw placements: accuracy and rates of cranial facet joint violation using conventional fluoroscopy compared with intraoperative three-dimensional computed tomography computer navigation. Eur Spine J 2016;25(06):1775-1780

9 Park P, Garton HJ, Gala VC, Hoff JT, McGillicuddy JE. Adjacent segment disease after lumbar or lumbosacral fusion: review of the literature. Spine 2004;29(17):1938-1944

10 Weinstein JN, Rydevik BL, Rauschning W. Anatomic and technical considerations of pedicle screw fixation. Clin Orthop Relat Res 1992;(284):34-46

11 Pathria M, Sartoris DJ, Resnick D. Osteoarthritis of the facet joints: accuracy of oblique radiographic assessment. Radiology 1987; 164(01):227-230
12 Stieber J, Quirno M, Cunningham M, Errico TJ, Bendo JA. The reliability of computed tomography and magnetic resonance imaging grading of lumbar facet arthropathy in total disc replacement patients. Spine 2009;34(23):E833-E840

13 Babu R, Park JG, Mehta AI, et al. Comparison of superior-level facet joint violations during open and percutaneous pedicle screw placement. Neurosurgery 2012;71(05):962-970

14 Chung KJ, Suh SW, Swapnil K, Yang JH, Song HR. Facet joint violation during pedicle screw insertion: a cadaveric study of the adult lumbosacral spine comparing the two pedicle screw insertion techniques. Int Orthop 2007;31(05):653-656

15 Chen Z, Zhao J, Xu H, Liu A, Yuan J, Wang C. Technical factors related to the incidence of adjacent superior segment facet joint violation after transpedicular instrumentation in the lumbar spine. Eur Spine J 2008;17(11):1476-1480

16 Jones-Quaidoo SM, Djurasovic M, Owens RK II, Carreon LY. Superior articulating facet violation: percutaneous versus open techniques. J Neurosurg Spine 2013;18(06):593-597

17 Kim HJ, Jung WI, Chang BS, Lee CK, Kang KT, Yeom JS. A prospective, randomized, controlled trial of robot-assisted vs freehand pedicle screw fixation in spine surgery. Int J Med Robot 2017;13 (3).

18 Matsukawa K, Kato T, Yato Y, et al. Incidence and risk factors of adjacent cranial facet joint violation following pedicle screw insertion using cortical bone trajectory technique. Spine 2016; 41(14):E851-E856

19 Moshirfar A, Jenis LG, Spector LR, et al. Computed tomography evaluation of superior-segment facet-joint violation after pedicle instrumentation of the lumbar spine with a midline surgical approach. Spine 2006;31(22):2624-2629

20 Shah RR, Mohammed S, Saifuddin A, Taylor BA. Radiologic evaluation of adjacent superior segment facet joint violation following transpedicular instrumentation of the lumbar spine. Spine 2003;28(03):272-275

21 Hailong Y, Wei L, Zhensheng M, Hongxun S. Computer analysis of the safety of using three different pedicular screw insertion points in the lumbar spine in the Chinese population. Eur Spine J 2007;16(05):619-623

22 Miekisiak G, Kornas P, Lekan M, Dacko W, Latka D, Kaczmarczyk J. Accuracy of the free-hand placement of pedicle screws in the lumbosacral spine using a universal entry point: clinical validation. J Spinal Disord Tech 2015;28(04):E194-E198

$23 \mathrm{Oh}$ CH, Yoon SH, Kim YJ, Hyun D, Park HC. Technical report of free hand pedicle screw placement using the entry points with junction of proximal edge of transverse process and lamina in lumbar spine: analysis of 2601 consecutive screws. Korean J Spine 2013;10(01):7-13

24 Archavlis E, Carvi y Nievas M. Comparison of minimally invasive fusion and instrumentation versus open surgery for severe stenotic spondylolisthesis with high-grade facet joint osteoarthritis. Eur Spine J 2013;22(08):1731-1740

25 Bastian L, Knop C, Lange U, Blauth M. [Transpedicular implantation of screws in the thoracolumbar spine. Results of a survey of methods, frequency and complications]. Orthopade 1999;28(08): 693-702

26 Wanibuchi M, Komatsu K, Akiyama Y, Mikami T, Mikuni N. Effectiveness of the 3D monitor system for medical education during neurosurgical operation. World Neurosurg 2018;109: e105-e109 